\title{
Right Bundle Branch Block by ECG Finding
}

National Cancer Institute

\section{Source}

National Cancer Institute. Right Bundle Branch Block by ECG Finding. NCI Thesaurus.

Code 62270.

An electrocardiog raphic finding of a wide QRS complex with evidence of delayed conduction to the right ventricle, manifested by a widened initial portion of the QRS in V1 and V2, a widened S wave in V5, V6, I and aVL, and with QRS duration greater than or equal to 120 ms. An RsR' complex is typically present in leads V1 and V2. (CDISC) 